\title{
Establishment of a Simple and Rapid Gene Delivery System for Cucurbits by Using Engineered Zucchini Yellow Mosaic Virus
}

\author{
Minji Kang ${ }^{1, \uparrow}$, Jang Kyun Seo ${ }^{2, \uparrow}$, Hoseong Choi ${ }^{1}$, Hong Soo Choi ${ }^{2}$ and Kook Hyung Kim ${ }^{1,3 *}$ \\ ${ }^{I}$ Department of Agricultural Biotechnology and Research Institute of Agriculture and Life Sciences, Seoul National Univer- \\ sity, Seoul 151-921, Korea \\ ${ }^{2}$ Crop Protection Division, National Academy of Agricultural Science, Wanju 565-852, Korea \\ ${ }^{3}$ Plant Genomics and Breeding Institute, Seoul National University, Seoul 151-921, Korea
}

(Received on August 28, 2015; Revised on September 30, 2015; Accepted on October 2, 2015)

The infectious full-length cDNA clone of zucchini yellow mosaic virus (ZYMV) isolate PA (pZYMV-PA), which was isolated from pumpkin, was constructed by utilizing viral transcription and processing signals to produce infectious in vivo transcripts. Simple rub-inoculation of plasmid DNAs of pZYMV-PA was successful to cause infection of zucchini plants (Cucurbita pepo L.). We further engineered this infectious cDNA clone of ZYMV as a viral vector for systemic expression of heterologous proteins in cucurbits. We successfully expressed two reporter genes including $g f p$ and $b a r$ in zucchini plants by simple rub-inoculation of plasmid DNAs of the ZYMV-based expression constructs. Our method of the ZYMVbased viral vector in association with the simple rubinoculation provides an easy and rapid approach for introduction and evaluation of heterologous genes in cucurbits.

Keywords : gene delivery, infectious clone, ZYMV

Zucchini yellow mosaic virus (ZYMV), which belongs to the genus Potyvirus, is a flexuous rod-shaped virus of about $750 \mathrm{~nm}$ in length and has a genome of a plus-sense singlestranded RNA of $9.5 \mathrm{~kb}$ (Gal-On et al., 1991). ZYMV is one of the major pathogens in cucurbitaceous crops worldwide (Lecoq et al. 2009; Simmons et al., 2013). ZYMV

\footnotetext{
${ }^{\dagger}$ Equally contributed.

*Corresponding author.

Phone) +82-2-880-4677, FAX) +82-2-873-2317

E-mail)kookkim@snu.ac.kr

This is an open access article distributed under the terms of the Creative Commons Attribution License (http://creativecommons.org/licenses/by/2.0), which permits unrestricted use, distribution, and reproduction in any medium, provided the original work is properly cited.
}

causes severe stunting and yellowing symptoms on leaves, and stream and deformation in fruits of cucurbitaceous crops (Kwon et al., 2005). ZYMV possesses a covalently linked viral protein (VPg) at the 5'-terminus and a poly (A) tail at the 3'-termius (Arazi et al., 2001). The genome of ZYMV encodes a large polyprotein and a putative pretty interesting potyviral protein (PIPO) at the $\mathrm{N}$ terminus of the P3 region (Chung et al., 2008). The large polyprotein is cleaved by self-encoded proteases into at least ten functional proteins, including $\mathrm{P} 1$, helper component protease (HC-Pro), $\mathrm{P} 3,6 \mathrm{~K} 1$, cylindrical inclusion protein (CI), 6K2, $\mathrm{VPg}$, NIa-Pro, NIb, and coat protein (CP) (Arazi et al., 2001; Kwon et al., 2005).

Cucurbitaceous crops, which belong to the family Cucurbitaceae, are an important part of diverse nutrition diet worldwide. The cucurbitaceous crops mostly cultivated in Korea include watermelon, cucumber, squash, and oriental melon (Kwon et al., 2005). However, research tools capable of introducing and evaluating valuable genes into cucurbitaceous crops are very limited. The plant virusbased vectors have been developed to express various foreign proteins in a wide range of host plants, since plant viruses accumulate high levels of virus-encoded proteins in infected plants (Gleba et al., 2014; Hefferon, 2012). The polyprotein expression system of potyviruses is advantageous to express foreign proteins as a part of viral proteins (Arazi et al., 2001; Seo et al., 2009). So far, various potyviruses, including soybean mosaic virus, turnip mosaic virus, tobacco etch virus, potato virus $A$, have been already engineered as vectors for expression of heterologous genes (Beauchemin et al., 2005; Dolja et al., 1992; Kelloniemi et al., 2008; Seo et al., 2009). Commonly, two sites between P1 and HC-Pro cistrons and between NIb and CP cistrons have been used to insert target genes in potyvirusbased vectors (Beauchemin et al., 2005; Kelloniemi et al., 2008). 
In this study, we constructed the infectious full-length cDNA clone of ZYMV and engineered it as a viral vector for systemic expression of heterologous proteins in cucurbits. Upon simple rub-inoculation of plasmid DNAs of the ZYMV-based viral vector, we successfully expressed two reporter genes including $g f p$ (the green fluorescence protein gene) and bar (the herbicide-resistance gene) in zucchini plants.

The ZYMV isolate PA (ZYMV-PA) obtained from pumpkins at Andong city (Kwon et al., 2005) was propagated in Cucumis melo L. (oriental melon) in a greenhouse and used for construction of infectious full-length cDNA clone of ZYMV. Total RNAs were extracted from the C. melo L. leaves infected with ZYMV-PA using the TRI Reagent (MRC, USA) according to the protocols provided by the manufacturer. The extracted total RNA was used for cDNA synthesis of ZYMV-PA. The fulllength cDNA clone of ZYMV-PA was constructed by assembling three partial amplified fragments of the ZYMV-PA cDNA. The first fragment including the ZYMV nucleotides from 1 to 1,807 (ZYMV 1-1807) was amplified by RT-PCR using specific primers (ZYPA-F, 5'-AAAATTGAAACAAATCACAAAGACTATAAG-3' and ZYPA-1807-BamHI-R, 5'-ACGGATCCAACT TACGGGTACCATTT-3'). The resulting amplicon was digested with BamHI and inserted into between the StuI and BamHI sites of pCass-RZ, which is a modified binary vector (de Wispelaere and Rao, 2009). The resulting clone was named pZYMVF1. The second fragment (ZYMV 1788-5938) was amplified by RT-PCR using specific primers (ZYPA-1788-F, 5'-CAAATGGTACCCGTAAGTTGG-3' and ZYPA5938-R, 5'-CCAGTGAGAGGATCCACAA-3'). The resulting product was digested with $K p n I$ and $B a m \mathrm{HI}$ and ligated with pZYMVF1, which was opened with KpnI and BamHI. The resulting clone was named pZYMVF1F2. For amplification of the last fragment (ZYMV 5911-9593), cDNA was synthesized using a tagged oligo(dT) primer (ZYPA-dTBamHI-RT, 5'-GCTGGGATCCGTGGTAAATTTTT TTTTTTTTTTTTTTTTTTTT-3') to hybridize to the ZYMV poly(A) tail. The cDNA was then amplified by PCR using specific primers (ZYPA-5911-F, 5'-CATCAGATTTGTGGATCCTCTC-3' and ZYPABamHI-R, 5'-GCTGGGATCCGTGGTAAA-3'). The resulting product was digested with $\mathrm{BamHI}$ and ligated with pZYMVF1F2, which was opened with BamHI. This final clone designated as pZYMV-PA contained full-length cDNA of ZYMV-PA with 25 adenines at the 3 ' end (Fig. 1).

To examine the infectivity of the cloned ZYMV-PA, purified plasmid DNAs of pZYMV-PA was inoculated onto the leaves of zucchini plants $(C$. pepo L.) by direct rub-inoculation with carborundum. Ten $\mu \mathrm{g}$ of $\mathrm{pZYMV-}$ PA prepared using the Plasmid Maxi Kit (QIAGEN, USA) was mixed with inoculation buffer $(50 \mathrm{mM}$ potassium phosphate, $\mathrm{pH} 7.5$ ) to a total volume of $80 \mu \mathrm{l}$ for the inoculation of two leaves per plant. At 12 days postinoculation (dpi), zucchini plants inoculated with pZYMVPA showed symptoms of yellowing, deformation, and mosaic, similar to those caused by the original ZYMV-PA (Fig. 2A). To confirm ZYMV infection of the inoculated plants, total RNAs extracted from the upper un-inoculated leaves were subjected to RT-PCR using the ZYMVspecific primers (5'-CTTTGAACAAGGAGACACTG-3' and 5'-GCGGTAAATATTAGAATTAC-3') that detect the CP region of ZYMV (Fig. 2B). A band of the predicted size (approximately $837 \mathrm{bp}$ ) was observed in all inoculated samples (Fig. 2C). Therefore, the results demonstrated that pZYMV-PA is fully infectious.

To develop pZYMV-PA as a viral vector for expres-

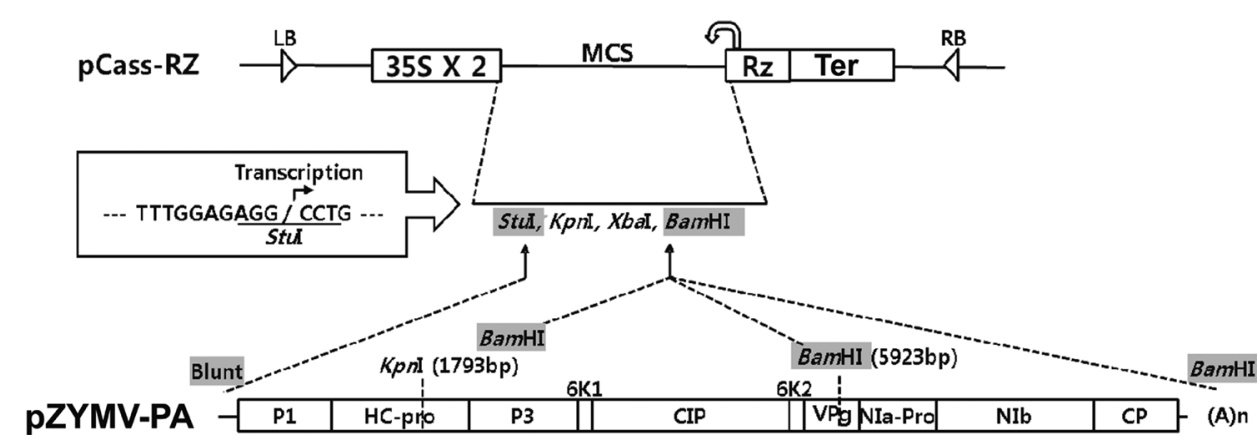

Fig. 1. Schematic representation of construction of the infectious full-length cDNA clone of zucchini yellow mosaic virus. The pCassRZ vector contains, in sequential order, a left border of T-DNA (LB), a double CaMV 35S promoter (35S X 2), a cis-cleaving ribozyme sequence (RZ), a 35S terminator (Ter), and a right border of T-DNA (RB). The restriction enzyme cleavage sites used to construct the full-length clone are shown in gray boxes. 

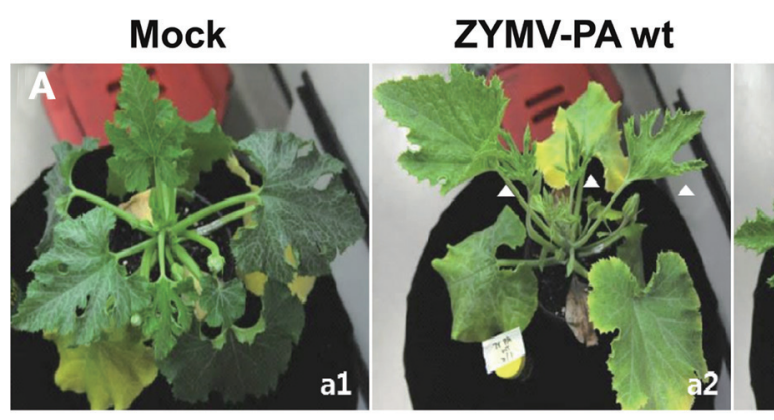

PZYMV-PA

B

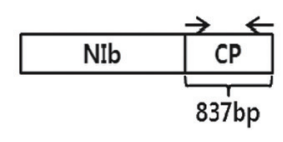

C

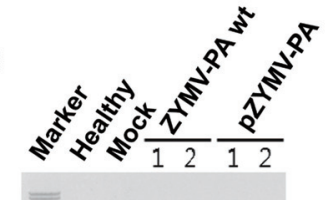

Fig. 2. Infection of zucchini plants upon mechanical rub-inoculation of plasmid DNAs of pZYMV-PA. (A) Wild-type (wt) virus of ZYMV-PA (ZYMV-PA wt) and the cloned ZYMV-PA (pZYMV-PA) were mechanically inoculated on the leaves of zucchini plants. At 12 days post inoculation (dpi), ZYMV-PA wt (a2) and pZYMV-PA (a3) caused same symptoms of yellowing, leaf deformation (arrowheads) and mosaic. (B) The primer pair (the marked arrows) spanning the CP region was used for RT-PCR detection of ZYMV. (C) Infectivity of pZYMV-PA was confirmed by RT-PCR detection of ZYMV replication. Arrow indicates PCR bands of the predicted size.

\section{A pZYMV-MCS}

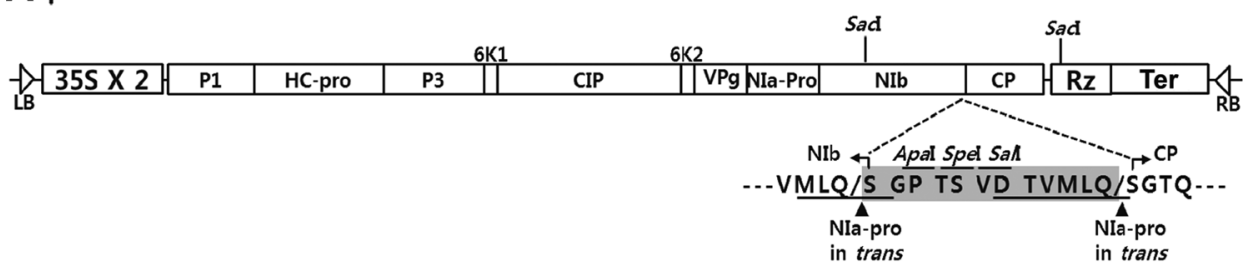

B

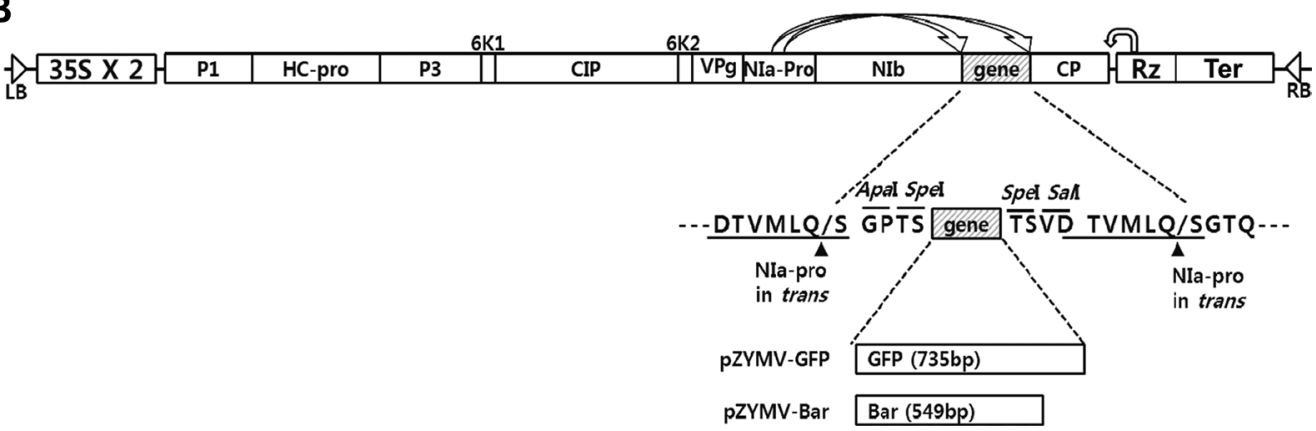

Fig. 3. Schematic representation of ZYMV-based viral vector constructs. (A) ZYMV genome organization and introduction of a gene insertion cassette between the NIb and $\mathrm{CP}$ cistrons. The shaded box indicates the gene insertion cassette containing multiple cloning sites (MCS: ApaI, SpeI, and SalI) and a NIa-Pro cleavage site (/S---DTVMLQ/). (B) Diagrammatic representation of insertion of the $g f p$ and $b a r$ genes into the ZYMV-based vector. The $g f p$ and bar genes were inframe-inserted into the pZYMV-MCS vector utilizing the SpeI site.

sion of heterologous genes in cucurbit plants, cloning sites (ApaI, SpeI and SalI) and an additional NIaPro cleavage site (/S---DTVMLQ/) were inserted into the polyprotein ORF between the NIb and CP cistrons (Fig. 3A). To this end, two SacI sites in pZYMV-PA were utilized (Fig. 3A). The NIb region downstream 


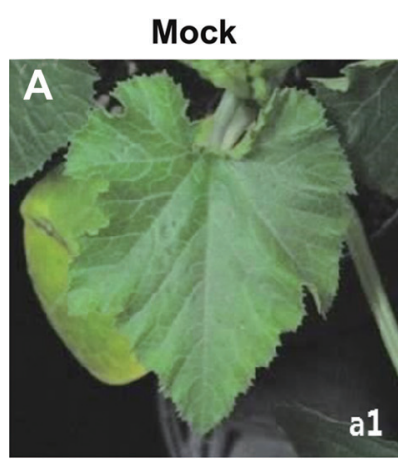

B

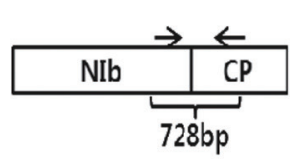

PZYMV-PA
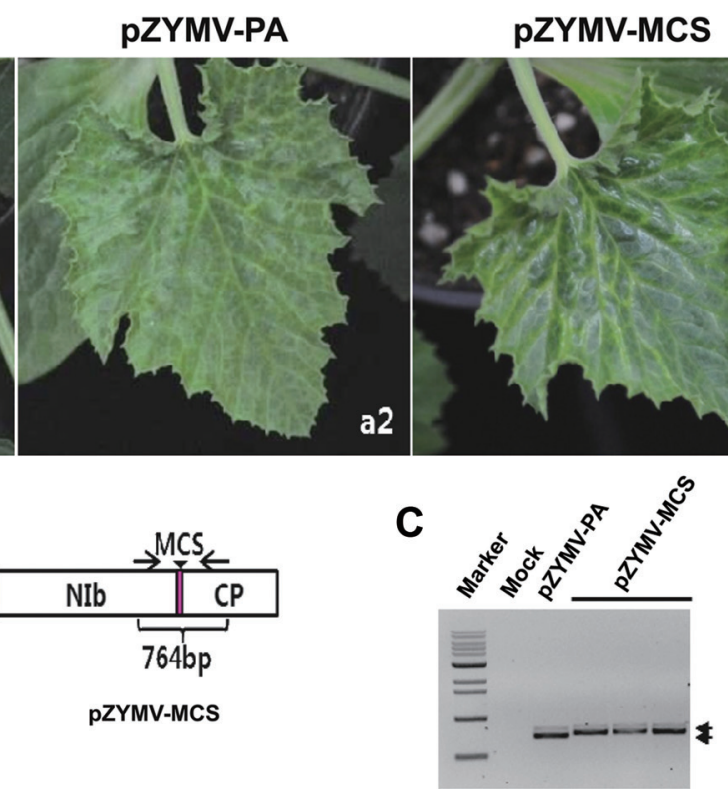

Fig. 4. Infection of zucchini plants upon mechanical rub-inoculation of plasmid DNAs of pZYMV-MCS. (A) Plasmid DNAs of pZYMV-PA and pZYMV-MCS were mechanically inoculated on the leaves of zucchini plants. At 12 dpi, pZYMV-PA wt (a2) and pZYMV-PA (a3) caused similar symptoms of yellowing, leaf deformation, and mosaic on zucchini plants. (B) The primer pair indicated by arrows was used for RT-PCR detection of ZYMV. (C) Infectivity of pZYMV-MCS was confirmed by RT-PCR detection of ZYMV replication. Arrows indicate PCR bands of the predicted sizes.

of the first $\mathrm{SacI}$ site was amplified using a primer pair (ZYPA-NIb-F, 5'-CCTGTGGAATGGATCTTTAA AGGCTGAGCTCAGAC-3' and ZYPA-NIb(NIa_MCS)-R, 5'-GACTGTCGACACTAGTGGGCCCCGACTGGAG CATCACAGTGTCTCCTTGTTCAAAGAAAATG-3'; the cloning sites are underlined) and subcloned into pGEM-T Easy Vector (Promega, USA). This intermediate clone was named pPA-MCS1-T. Next, the CP region upstream of the second $S a c$ I site was amplified using a primer pair (ZYPA-(MCS_NIa)CP-F, 5'-AGTCGTCGACACTGTGATGCTCC $\bar{C}$ ATCGG GCACTCAGCAAACTGTGG-3' and pCass-SalIR, 5'-GACTGTCGACTGATTTCAGCGTACCGAA TTCGAGCTC-3') and digested with SalI. The resulting fragment was inserted into pPA-MCS1-T that was opened with SalI. The resulting clone was named pPA-MCS2$\mathrm{T}$. Then, the ZYMV NIb/CP region harboring the cloning sites and a NIa-Pro cleavage site was cut out from pPAMCS2-T by digestion with SacI and inserted into pZYMVPA, which was opened with SacI. This final clone was named pZYMV-MCS (Fig. 3A). The infectivity of pZYMV-MCS was investigated by mechanical inoculation of zucchini plants using purified plasmid DNAs of pZYMV-MCS as described above. At 12 dpi, zucchini plants inoculated with pZYMV-MCS showed similar disease symptoms when compared with those induced by
pZYMV-PA (Fig. 4A). Infection of the inoculated plants with ZYMV conformed by RT-PCR using the primer pair (5'-CCATCAAGCAATGTTGGTTGAT-3' and 5'-CGACTCCCATCTGTTGATCATT-3') that detects the $\mathrm{NIb} / \mathrm{CP}$ region (Fig. 4B and $\mathrm{C}$ ).

To evaluate the expression of foreign genes from the ZYMV vector, two reporter genes, $g f p$ and bar, were amplified by PCR using appropriate primers (GFP-SpeI-F, 5'-GACTAGTATGCATCATCATCATCATCATGTG-3' and GFP-SpeI-R, 5'-GACTAGTCTTGTACAGCTCG TCCATGC-3' for $g f p$; Bar-SpeI-F, 5'-GACTAGTATG AGCCCAGAACGACGC-3' and Bar-SpeI-R, 5'-GACTA GTAATCTCGGTGACGGGCAG-3' for bar) and inserted into pZYMV-MCS utilizing the SpeI cloning site. The resulting constructs were designated as pZYMV-GFP and pZYMV-Bar (Fig. 3B). The plasmid DNAs of pZYMVGFP and -Bar were inoculated onto the cotyledons of zucchini seedlings by direct rub-inoculation. At $12 \mathrm{dpi}$, both of the constructs induced typical yellowing and mosaic symptoms of ZYMV (data not shown). In addition, strong signals of green fluorescence emitted by GFP were observed on the systemic leaves of the plants inoculated with pZYMV-GFP when the plants were visualized under illumination with a hand-held UV-light source (Dark Reader Hand Lamp HL28T; Clare Chemical Research, Dolores, CO) (Fig. 5). At 12 dpi, the zucchini plants infected by 

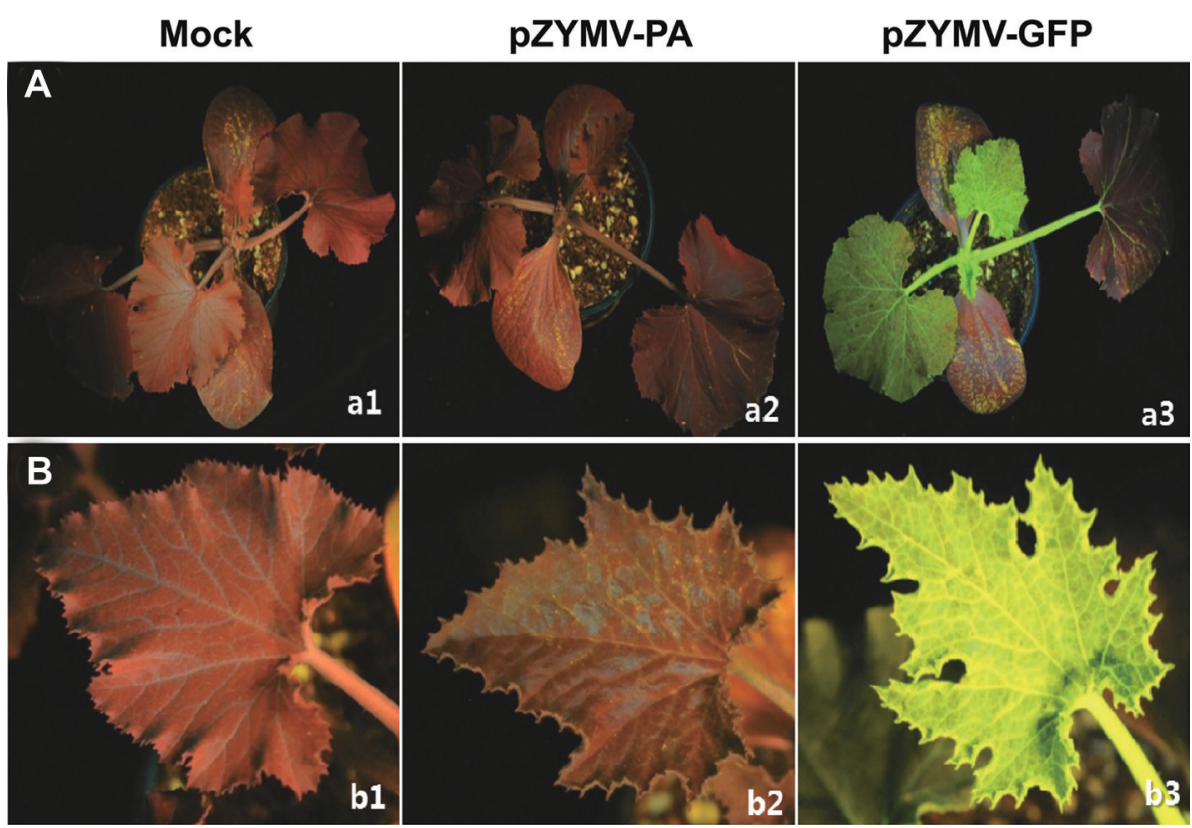

Fig. 5. Detection of fluorescence signals emitted by GFP under UV light on leaves of zucchini plants inoculated with pZYMV-GFP. (A) Plasmid DNAs of pZYMV-PA and pZYMV-GFP were mechanically inoculated on the leaves of zucchini plants. At 7 dpi, systemic leaves of zucchini plants inoculated with pZYMV-GFP (a3) showed intense fluorescence. No fluorescence was detected on leaves of healthy (a1) and pZYMV-PA-inoculated plants (a2). (B) The closed view of the systemic leaves photographed under UV light at 12 dpi.

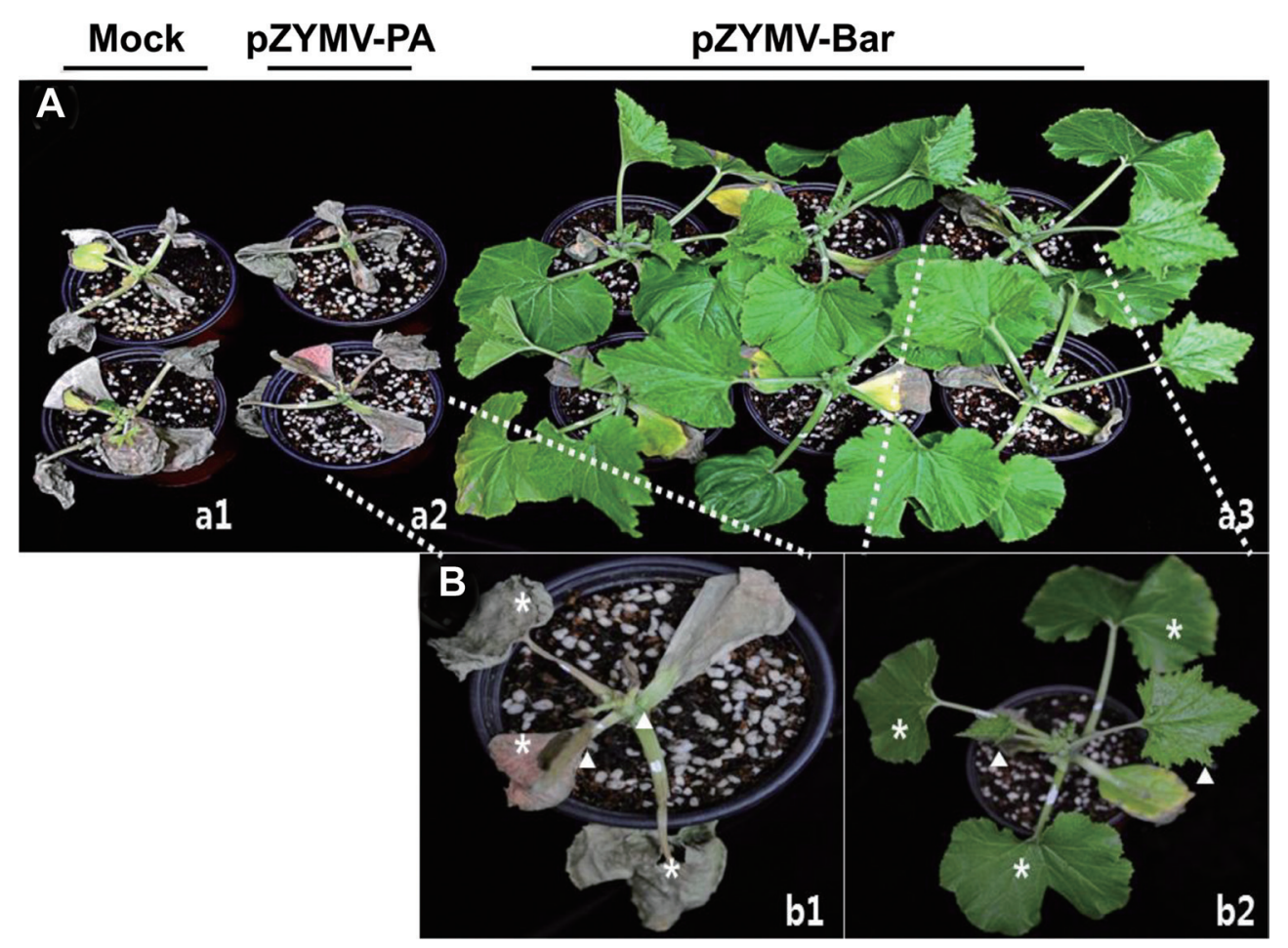

Fig. 6. Acquiring herbicide resistance of zucchini plants infected by pZYMV-Bar. The zucchini plants were treated with a non-selective herbicide $(0.54 \%$ glufosinate-ammonium $(\mathrm{w} / \mathrm{v}))$ at 12 day post inoculation of the indicated inoculums. (A) The representative photographs of the zucchini plants inoculated with pZYMV-PA (a2) and pZYMV-Bar (a3) at 7 days after herbicide treatment. (B) Magnified images of the zucchini plants inoculated with pZYMV-PA (b1) and pZYMV-Bar (b2). Asterisks indicate leaves treated with herbicide and arrowheads indicate upper leaves that grew after herbicide treatment. 

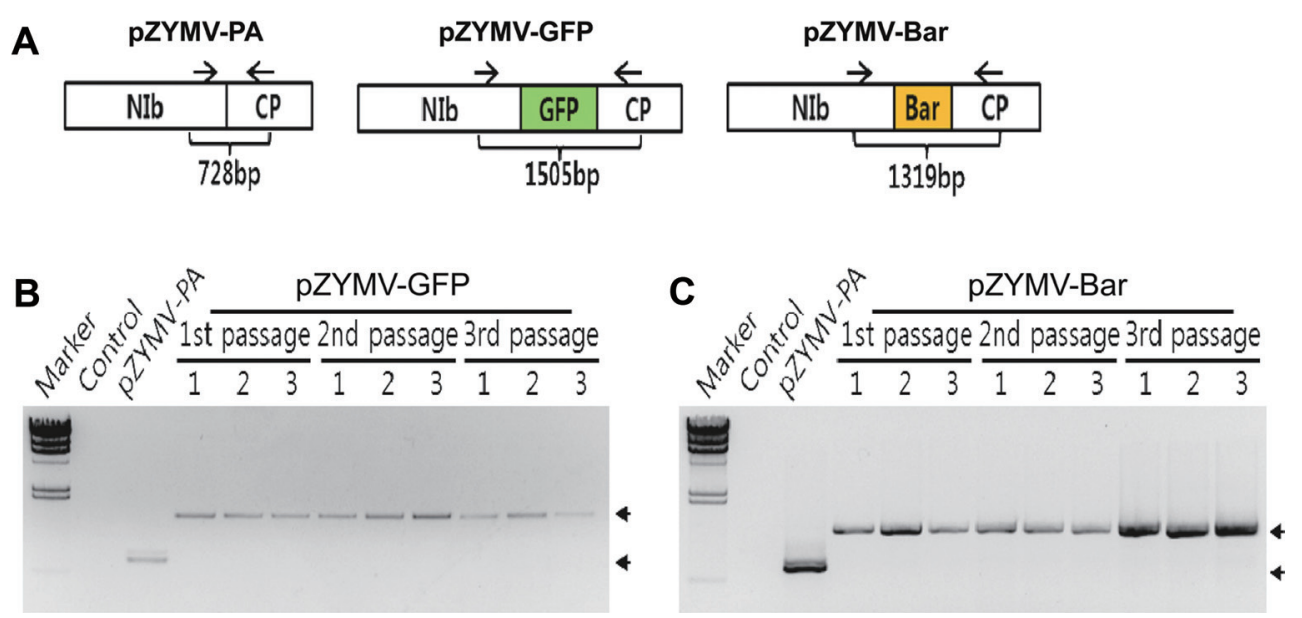

Fig. 7. Analysis of stability of the reporter genes in the ZYMV genome. (A) Schematic maps of the NIb/CP, NIb/GFP/CP and NIb/Bar/ $\mathrm{CP}$ regions. Arrows indicate the region that has been amplified by RT-PCR. (B and C) Total RNAs were extracted from upper systemic leaves of healthy, pZYMV-PA-infected, pZYMV-GFP-infected, or pZYMV-Bar-infected zucchini plants and subjected to RT-PCR. Progeny viruses in plant sap inoculated with either pZYMV-GFP or pZYMV-Bar were passaged three times to new plants. The upper arrows indicate the PCR products containing the gfp or bar gene, respectively. Arrows indicate PCR bands of the predicted sizes.

pZYMV-Bar were treated with a non-selective herbicide $(0.54 \%$ glufosinate-ammonium (w/v)). At 7 days after herbicide treatment, while the healthy and pZYMV-PAinoculated plants were significantly damaged, the plants inoculated with pZYMV-Bar were still healthy, indicating the expression of bar gene from the ZYMV viral vector is sufficient to confer herbicide resistance in zucchini plants (Fig. 6).

To evaluate the stability of the foreign gene insertion in the ZYMV viral vector, viruses obtained from the plant infected with either pZYMV-GFP or -Bar were passaged three times from plant to plant by mechanical sapinoculation. Total RNA was extracted from upper systemic leaves of each individual plant and analyzed for stable gene insertion in the viral genome by RT-PCR using the primer pair (5'-CCATCAAGCAATGTTGGTTGAT-3' and 5'-CGACTCCCATCTGTTGATCATT-3') that detects the $\mathrm{NIb} / \mathrm{CP}$ region (Fig. 7A). A unique band with the predicted size (approximately $1.5 \mathrm{kbp}$ for $g f p$ insertion and $1.3 \mathrm{kbp}$ for bar insertion) was observed for every plants infected by either pZYMV-GFP or -Bar (Fig. 7B and C). The results suggest that foreign genes inserted in the ZYMV viral vector could be maintained stably during serial passages.

With recent advances in plant biotechnology, plants have been targeted to be engineered as a mass production system for recombinant proteins (Hefferon, 2012). The interest in using plants as a cost effective means for the production of valuable proteins has led to the development of various plant virus-based vectors (Gleba et al., 2014; Hefferon, 2012). Virus-mediated gene expression in plant is a superi- or system to the transgenic approach because plant viruses systemically infect plants and accumulate large quantities of viral proteins. Thus, various plant virus-based gene delivery vectors have been developed for the overproduction of recombinant proteins within a short period of time in plants (Gleba et al., 2014; Hefferon, 2012). Since the turnaround time for introduction of new traits into plants is short, the plant virus-based gene delivery system can facilitate large-scale analysis of gene functions based on gain-offunction studies.

In this study, we have developed the ZYMV-based gene delivery vector for efficient expression of heterologous proteins in cucurbit plants. When using potyvirus-based vectors, multiple foreign proteins can be expressed from one construct by utilizing the different genomic regions (for example, two sites between P1 and HC-Pro and NIb and $\mathrm{CP}$ cistrons for insertion of foreign genes (Beauchemin et al., 2005; Kelloniemi et al., 2008). A previous study has developed a bombardment-based gene expression system by engineering ZYMV as a viral vector (Arazi et al., 2001). However, in general, bombardment-based methods require expensive devices and materials and are less-efficient. On the other hand, we established a simple, easy and low cost method for inoculation of the ZYMV-based viral vector by direct rub-inoculation of purified plasmid DNAs of the vector. To this end, we utilized viral transcriptions and processing signals, including the CaMV $35 \mathrm{~S}$ promoter, a self-cleaving ribozyme sequence and a poly(A) signal, to produce infectious in vivo transcripts resembling the native ZYMV genomic RNA. This DNA-mediated rub-inocula- 
tion method has an advantage of not requiring expensive and complicated steps or special equipments to deliver a viral vector containing foreign genes. It is highly desirable to identify and evaluate valuable traits for improving productivity, environmental and disease resistance, and the commercial value of cucurbit plants. The availability of the ZYMV-based gene delivery system developed in this study will be helpful to begin rapid in planta screening and evaluation of valuable candidate genes.

\section{Acknowledgments}

This work was supported in part by grants from the Agenda Programs (PJ00922904 \& PJ01130602) funded by the Rural Development Administration and the Vegetable Breeding Research Center (No. 710001-07-05) through the Agriculture Research Center program from the Ministry for Food, Agriculture, Forestry and Fisheries, Republic of Korea. MK was supported by research fellowship from the Ministry of Education, Science, and Technology through the Brain Korea 21 Project.

\section{References}

Arazi, T., Slutsky, S. G., Shiboleth, Y. M., Wang, Y., Rubinstein, M., Barak, S., Yang, J. and Gal-On, A. 2001. Engineering zucchini yellow mosaic potyvirus as a non-pathogenic vector for expression of heterologous proteins in cucurbits. J. Biotechnol. 87:67-82.

Beauchemin, C., Bougie, V. and Laliberte, J. F. 2005. Simultaneous production of two foreign proteins from a polyvirusbased vector. Virus Res. 112:1-8.

Chung, B. Y., Miller, W. A., Atkins, J. F. and Firth, A. E. 2008. An overlapping essential gene in the Potyviridae. Proc. Natl. Acad. Sci. USA 105:5897-5902. de Wispelaere, M. and Rao, A. L. 2009. Production of cucumber mosaic virus RNA5 and its role in recombination. Virology 384:179-191.

Dolja, V. V., Mcbride, H. J. and Carrington, J. C. 1992. Tagging of plant potyvirus replication and movement by insertion of $\beta$-glucuronidase into the viral polyprotein. Proc. Natl. Acad. Sci. USA 89:10208-10212.

Gal-On, A., Antignus, Y., Rosner, A. and Raccah, B. 1991. Infectious in vitro RNA transcripts derived from cloned cDNA of the cucurbit potyvirus, zucchini yellow mosaic virus. J. Gen. Virol. 72:2639-2643.

Gleba, Y. Y., Tuse, D. and Giritch, A. 2014. Plant viral vectors for delivery by Agrobacterium. Curr. Top Microbiol. Immunol. 375:155-192.

Hefferon, K. L. 2012. Plant virus expression vectors set the stage as production platforms for biopharmaceutical proteins. Virology 433:1-6.

Kelloniemi, J., Makinen, K. and Valkonen, J. P. 2008. Three heterologous proteins simultaneously expressed from a chimeric potyvirus: infectivity, stability and the correlation of genome and virion lengths. Virus Res. 135:282-291.

Kwon, S. W., Kim, M. S., Choi, H. S. and Kim, K.-H. 2005. Biological characteristics and nucleotide sequences of three Korean isolates of zucchini yellow mosaic virus. J. Gen. Plant Pathol. 71:80-85.

Lecoq, H., Wipf-Scheibel, C., Chandeysson, C., Le Van, A., Fabre, F. and Desbiez, C. 2009. Molecular epidemiology of zucchini yellow mosaic virus in France: an historical overview. Virus Res. 141:190-200.

Seo, J. K., Lee, H. G. and Kim, K.-H. 2009. Systemic gene delivery into soybean by simple rub-inoculation with plasmid DNA of a soybean mosaic virus-based vector. Arch. Virol. 154:87-99.

Simmons, H. E., Dunham, J. P., Zinn, K. E., Munkvold, G. P., Holmes, E. C. and Stephenson, A. G. 2013. Zucchini yellow mosaic virus (ZYMV, Potyvirus): vertical transmission, seed infection and cryptic infections. Virus Res. 176:259-264. 